\title{
Behavioral Improvements and its Molecular Mechanism of Ilex kudingcha C.J. Tseng on Animal Model of Alzheimer's Disease
}

\author{
Lap Thi Nguyen ${ }^{1 *}$, Nguyen Huu Son ${ }^{1,2}$, Tran Nguyen Hong², Nguyen Minh Khoi ${ }^{2}$, Kinzo Matsumoto ${ }^{3}$, \\ William R. Folk ${ }^{4}$, Masamitsu Yamaguchi' ${ }^{5}$ Pham Thi Nguyet Hang² \\ ${ }^{1}$ Hanoi University of Pharmacy, Hanoi,Vietnam \\ ${ }^{2}$ National Institute of Medicinal Materials, Hanoi, Vietnam \\ ${ }^{3}$ Institute of Natural Medicine, University of Toyama, Japan \\ ${ }^{4}$ University of Missouri, USA \\ ${ }^{5}$ Kyoto Institute of Technology, Japan
}

\begin{abstract}
Alzheimer's disease (AD) is a common chronic neurodegenerative disease with well-defined pathophysiological mechanisms. Ilex kudingcha (IK) C.J. Tseng is commonly known as bitter tea or "Khom" tea in Vietnam. The present study was conducted to investigate the anti-dementia effect of IK using olfactory bulbectomized (OBX) mice. OBX mice were daily treated with IK extract $(540 \mathrm{mg} / \mathrm{kg})$ or reference drug, tacrine $(2.5 \mathrm{mg} / \mathrm{kg}) 1$ week before and continuously for 3 days after the OBX surgery. The object recognition test, modified Y maze test and fear conditioning test were employed to analyze non-spatial short-term, spatial short-term and long-term memories of the mice respectively. Administration of IK extract and tacrine attenuated these OBX-induced cognitive deficits in mice. The effects of IK and tacrine on spatial short-term memory impairment were reversed by scopolamine, a muscarinic receptor antagonist. The amyloid-beta $(\mathrm{A} \beta)$ production in adult transgenic Drosophila brain flies was also investigated by using Western blotting with APP-HA antibody. These results indicated that IK extract improves short-term and long-term memory disturbances in OBX mice and that muscarinic receptor may play a role on these actions. In addition, our result also showed that IK extract reduces the expression of amyloid precursor protein (APP) in brain of AD model using Drosophila melanogaster.
\end{abstract}

Key words: Alzheimer's disease; amyloid-beta; Drosophila models; Ilex kudingcha (IK) C.J. Tseng.

\section{INTRODUCTION}

Alzheimer disease (AD) is a neurodegenerative disease usually of a chronic or progressive nature which distinctively shows deterioration of cognitive function and the impaired ability to perform daily activities. Importantly, symptoms of dementia develop gradually and they typically become worse over a number of years. Since dementia has significant social and economic implications in terms of medical costs, social costs and the costs of informal care, development of new drugs or strategies which are not only effective for prevention/therapy of dementia but also capable of being taken for a long period without causing adverse reactions, are greatly needed.

IK known as bitter tea is indigenous to Limestone Mountains in Vietnam, distributed in some provinces such as Lao Cai, Lang Son, Cao Bang, Hoa Binh and Ninh Binh. In traditional Vietnamese medicine, IK has been used to resolve toxin, antibacterial, alleviate thirst and cough, itchy eyes, red eyes, especially to improve mental focus

\section{*Corresponding author : Lap Thi Nguyen}

Email : lapnt@hup.edu.vn and memory. Triterpenoids, phenolic acids, flavonoids, and essential oils are the main metabolites of IK an these ingredients protect the vascular system, regulate lipid metabolism, and have antioxidant, hypoglycemic, and anti-tumor effects [1]. In addition, Kim et al., reported that IK protects neuronal cell against transient focal ischemia-induced neuronal damage in rats [2]. Moreover, this herb was reported to exert neuroprotective effects against amyloid $\beta$ protein $(A \beta)$-induced memory impairment in mice [3] and neurotoxicity in cultured rat cortical neurons [4]. In this study, we clarified the beneficial effects of IK leaf extract in memory impairment induced by olfactory bulbectomy in mice. We also initially study the molecular mechanism by evaluating the expression level of the APP in AD model using Drosophila melanogaster.

\section{METHODOLOGY \\ Materials}

$I K$ leaf extract: IK leaves were collected in Cao Bang province, Vietnam. The plant samples were identified by Dr. Pham Thanh Huyen, Department of Resource Medicinal Materials, 


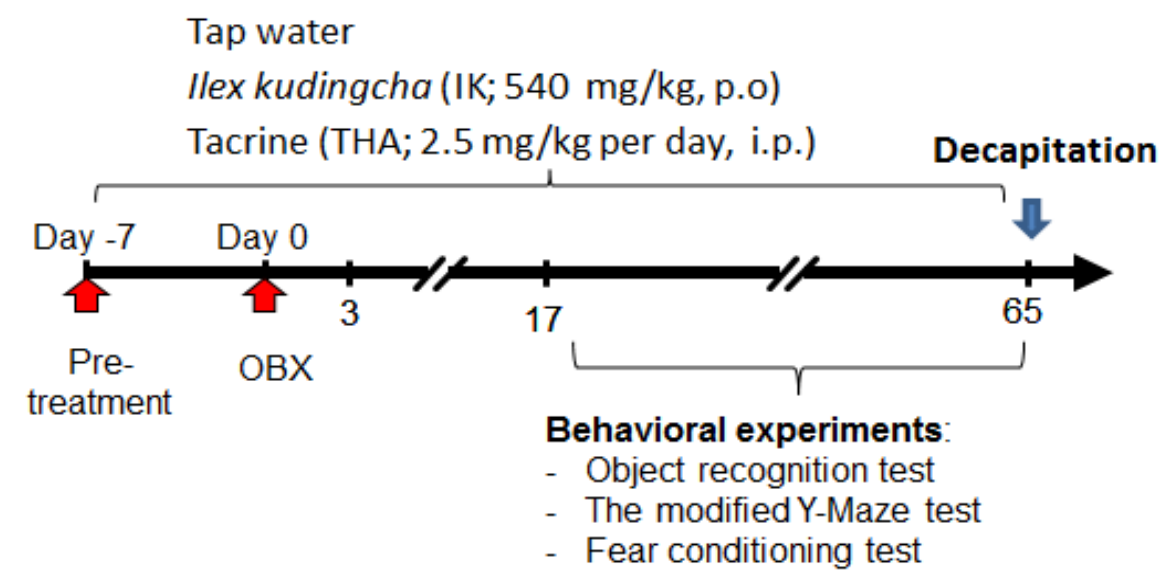

Figure 1. Experimental schedule

National Institute of Medicinals Materials. The leaves of IK were dried in a hot-air oven at 50 ${ }^{\circ} \mathrm{C}$ then crushed. The herbal powder was soaked in $80 \%$ alcohol $(1: 10 \mathrm{w} / \mathrm{v})$ for 48 hours. After filtration, the herbal material was repeatedly extracted with alcohol for 24 hours (2 times). The combined aqueous alcohol extract was concentrated under reduced pressure at $50^{\circ} \mathrm{C}$ until obtain a ratio of 1 : $2(\mathrm{w} / \mathrm{v})$. After being cooled down to room temperature, the extract was filtered. The filtrate was collected then dried in vacuum oven at $60^{\circ} \mathrm{C}$ until obtain the dry IK extract. Yield of extraction from the dried herb was calculated as $10.7 \%$.

\section{Animals:}

Male ddY mice (Japan SLC Inc., Shizuoka, Japan) were obtained at the age of 9 weeks old. The animals were habituated to the laboratory animal room for at least 1 week before surgery. Food and water were available ad libitum. Housing was thermostatically maintained at $24 \pm 1{ }^{\circ} \mathrm{C}$ with constant humidity (65\%) and a 12-h light-dark cycle (lights on: 07:00 - 19:00). The behavioural experiments were performed during the light phase from 9:00 to 18:00.

Two fruit fly lines provided by Prof Masamitsu Yamaguchi, Kyoto Institute of Technology, Japan: hAPP.HA (BL64385) and Elav Gal4 (III).

\section{Methods \\ OBX operation}

OBX operation was conducted as previously described with slight modification [5-7]. Briefly, mice were anesthetized with sodium pentobarbital (60 mg/kg, i.p.), and fixed on stereotactic instruments (Narishige, Tokyo, Japan). Skin incision was made to expose the skull covering the bulbs and then a $1 \mathrm{~mm}$ burr hole was drilled. The bilateral bulbs were aspired through a syringe and the cavity of the bulbs was filled with hemostatic gelatin sponge. After completing the behavioural studies, all the animals were sacrificed to verify the operated lesion visually. The data from animals with less than $70 \%$ removal or with no intact cortex were excluded from the analysis. Sham operation was performed in a similar way without removal of the bulb. At the end of the experiments, the olfactory bulbs of sham group mice were confirmed to be intact.

\section{Drug administration}

Animals received daily administration of IK extract ( $540 \mathrm{mg} / \mathrm{kg}$, p.o) or tacrine $(2.5 \mathrm{mg} / \mathrm{kg}$, i.p) or tap water for 1 week before OBX surgery and these treatments were re-started from day 3 after the operation (Figure 1). Sham group of mice and OBX control group mice were per orally administered water. Reference standard drug, tacrine (THA; 9-amino-1,2,3,4-tetrahydro-acridine $\mathrm{HCl}$, Sigma, St. Louis) was dissolved in $0.9 \%$ saline. The IK extract was suspended in water. On a behavioural testing day, administration was conducted $1 \mathrm{~h}$ before the testing.

\section{Novel object recognition test (ORT)}

ORT was conducted to clarify the effect of IK extract on object recognition memory (non-spatial short-term memory) on day 17 (Figure 1) as previously reported $[6,7]$. ORT is based on the tendency of mice to discriminate a familiar from a new object. One day before the test, mice were individually habituated to an open-field box $(35 \mathrm{x}$ $35 \times 50 \mathrm{~cm}$ ) for $10 \mathrm{~min}$. The ORT consists of a sample phase trial and a test phase trial. During the sample phase trial, two objects of the same material were placed in a symmetric position in the 
A)

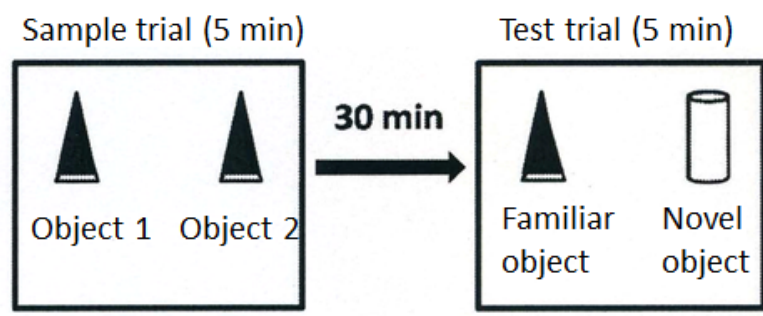

B) Sample trial

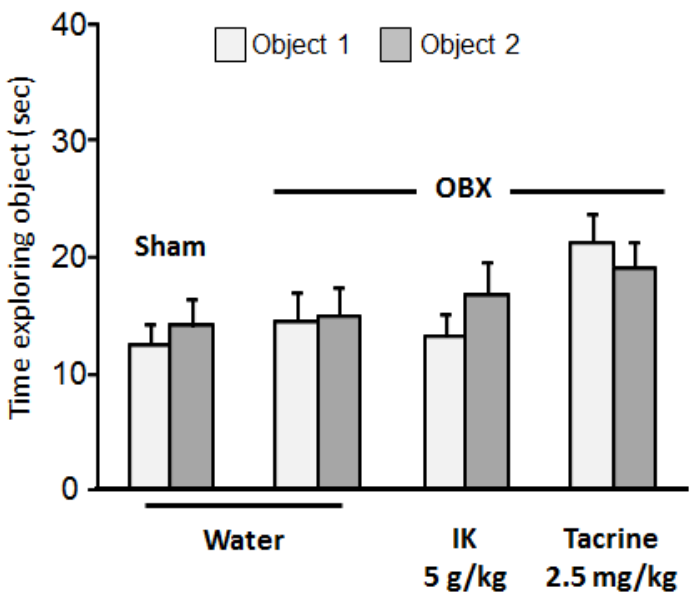

C) Test trial

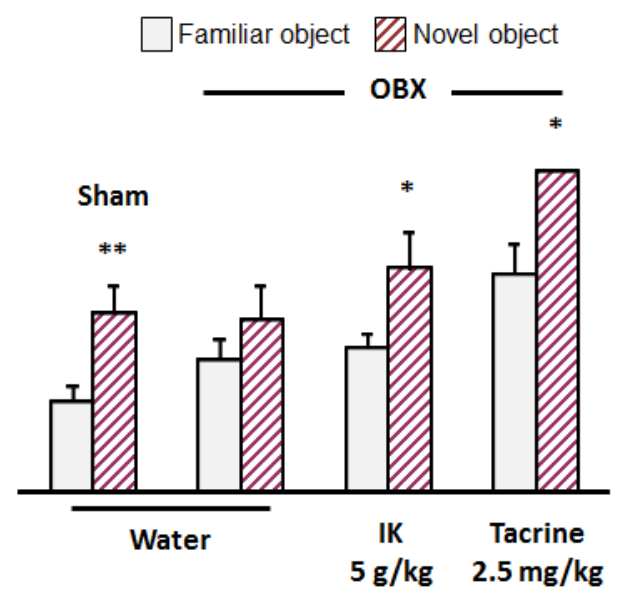

Figure 2. Effects of IK extract on OBX-induced object recognition memory deficit in mice. ${ }^{*} \mathrm{P}<0.05$, ${ }^{*} \mathrm{P}<0.01$ vs. family object $(\mathrm{n}=9-12)$

centre of the chamber for 5 min. Thirty minutes after the sample phase trial, one of the objects was replaced by a novel object, and exploratory behaviour was analyzed for 5 min (Figure 2A). After each session, objects were thoroughly cleaned with $70 \%$ ethanol to prevent recognition of odor. Exploration of an object was defined as rearing on the object or sniffing it at a distance of less than $2 \mathrm{~cm}$. Successful recognition of a previously explored object was reflected by preferential exploration of the novel object. Discrimination of object novelty was assessed by comparing the difference between time of exploration of the novel and familiar objects. Thetime spent exploring each of the two objects wasanalyzed using SMART ${ }^{\circledR}$ system ver. 2.5 with a tri-wise module to detect the head, center mass andbase-tail (PanLab, S.L., Barcelona, Spain).

\section{Modified Y-maze test}

The modified Y-maze test was performed at day 24 and 31 after OBX surgery (Figure 1) previously described $[6,7]$. The apparatus used for this test consists of black polypropylene walls with $3 \mathrm{arms}$ each $40 \mathrm{~cm}$ long, $12 \mathrm{~cm}$ wide at the top, 3 $\mathrm{cm}$ wide at the bottom and $18 \mathrm{~cm}$ high (Figure $3 \mathrm{~A}$ ). This test consists of two-trial task with a sample phase trial and a test phase trial that were separated by an inter-trial interval. In the sample phase trial, each mouse was individually placed in the maze with one of the 3 arms closed. The mice were allowed to explore the other 2 arms freely for $5 \mathrm{~min}$. Thirty min after the sample phase trial, the animal was again placed in the maze with all 3 arms opened, and allowed to explore the arms freely. The previously closed arm that was opened in the test phase trial was defined as the new arm. The animal behavior was video-recorded for later analysis. Percent time spent in the new arm and numbers of total arm entries were analyzed using SMART ${ }^{\circledR}$ system ver. 2.5 (PanLab, S.L., Barcelona, Spain). In the $Y$ maze test at day 31, scopolamine $\mathrm{HBr}$ (Sigma, St. Louis, MO, USA) was dissolved in $0.9 \%$ saline and administered to IK and tacrinetreated OBX mice at a single dose of $1 \mathrm{mg} / \mathrm{kg}$ (i.p) $1 \mathrm{~h}$ before the test.

\section{Fear conditioning test}

The fear conditioning test was conducted to measure long-term memory in OBX mice 3 weeks after the modified Y maze test (day 52, Figure 1). This test was conducted according to the previously described method [7] with slight modification. Briefly, the chamber for fear conditioning consisted of a transparent acrylic 
A)

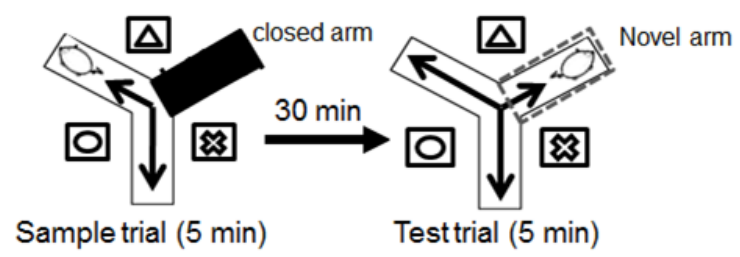

B)

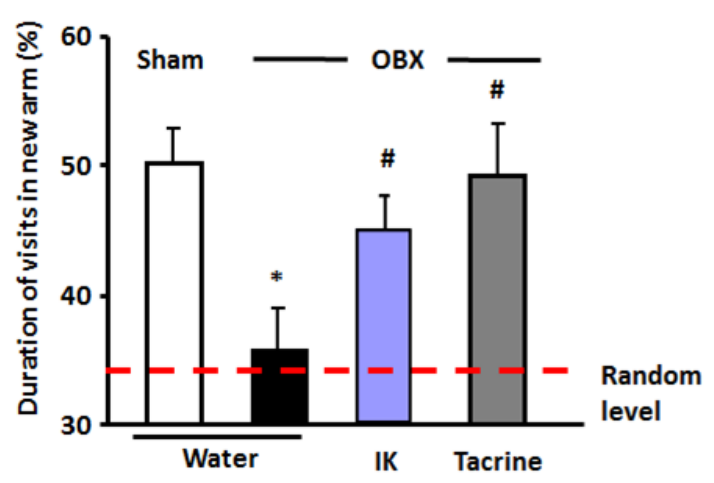

C)

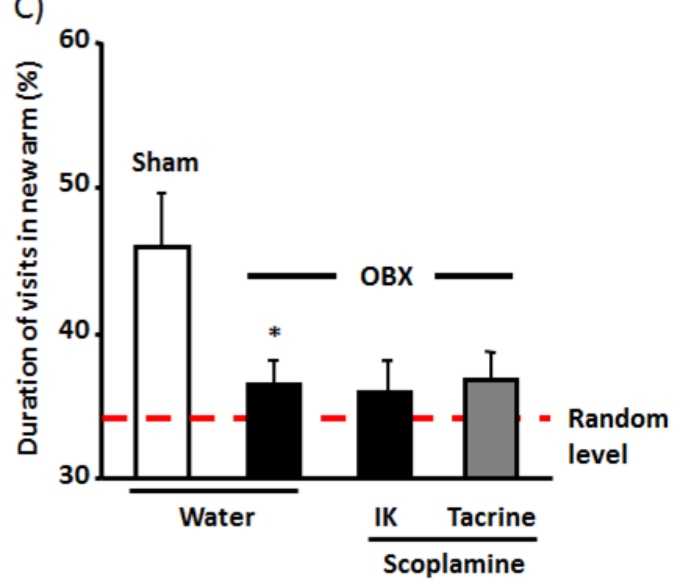

Figure 3. Effects of IK extract on spatial working memory in $\mathrm{OBX}$ mice. ${ }^{*} \mathrm{P}<0.05$ vs. sham group; \#P<0.05 vs. water-treated OBX group $(n=9-12)$

chamber $(30 \times 30 \times 30 \mathrm{~cm})$ and a stainless-steel grid floor equipped with an electric shock generator/scrambler SGS-002R, CS Controller CSS001R, and Cycle TimerCMTR (Muromachi Kikai. Co. Ltd., Tokyo, Japan). The apparatus was placed in a sound-proof observation box (MC-050/CM, Muromachi Kikai, Co. Ltd., Tokyo, Japan) through which the auditory tone $(2.9 \mathrm{kHz}, 80 \mathrm{~dB})$ (SonalertR, Mallory Sonalert Products Inc., Indianapolis, IN, USA) was delivered to the animal. In the training sessions, the animals were placed individually into the fear conditioning chamber and allowed to explore freely for $4 \mathrm{~min}$. Then, they received an acoustic tone $(2.9 \mathrm{kHz}, 20 \mathrm{~s}, 80 \mathrm{~dB}) \mathrm{co}-$ terminated with electric footshocks $(0.8 \mathrm{~mA}, 2 \mathrm{~s})$. The tone-footshock pairing was continuously repeated 5 times with a 1 -min interval. One minute after the final footshock delivery, the mice were returned to their home cage. The test sessions were conducted to measure freezing responses to the context and auditory tone 5 days after the training. The mice were placed in the same chamber to provide the contextual stimuli and allowed to move freely for $6 \mathrm{~min}$. Behavioral responses recorded for $3 \mathrm{~min}$ from $1 \mathrm{~min}$ after the animal was placed in the chamber were analyzed to measure freezing behavior as an index of contextual memory. After the contextual recording, the mice were exposed to the tone for 2 min, and freezing behavior during the 2-min period was recorded as auditory-dependent fear memory. The animal behavior was video-recorded and analyzed automatically by Smart® system. Freezing was defined as the absence of any movement except for those related to respiration. Footshock-naive mice were used as controls that likely exhibit pseudo-positive freezing response.

\section{Protein extraction and APP expression level assessment}

Total proteins from adult heads of flies carrying UAS-hAPP-HA treated with vehicle (water), 2 and $4 \mathrm{mg} / \mathrm{mL}$ of IK extract were extracted by using $2 x$ SDS sample buffer (2\% SDS, $10 \%$ glycerol, $0.002 \% \mathrm{BPB}$, and $0.063 \mathrm{M}$ Tris- $\mathrm{HCl}$ ), heated at $95^{\circ} \mathrm{C}$ for $2 \mathrm{~min}$, followed by centrifugation at $14000 \times \mathrm{g}$ at $4^{\circ} \mathrm{C}$ for $20 \mathrm{~min}$. Protein extracts were further separated by SDSpolyacrylamide gel electrophoresis containing $10 \%$ acrylamide (SDS-PAGE) and then transferred to polyvinylidene difluoride (PVDF) membranes (Merck, Millipore, MA, USA). The blotted membranes were blocked by Tris-buffered saline $/ 0.05 \%$ Tween 20 containing 5\% skim milk at $25^{\circ} \mathrm{C}$ for $1 \mathrm{~h}$ and then incubated at $4^{\circ} \mathrm{C}$ for $16 \mathrm{~h}$ with the proper primary antibodies: rabbit anti-HA IgG (1:1000, Sigma-Aldrich) and mouse anti-Actin IgG (ACTA2,1:1000, DSHB \#JLA20). After the 
A)
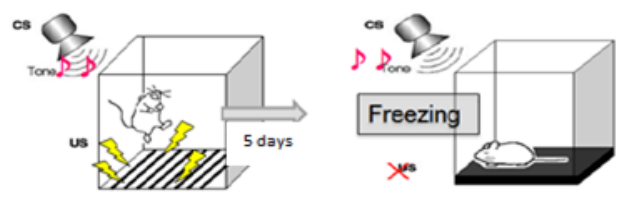

B)

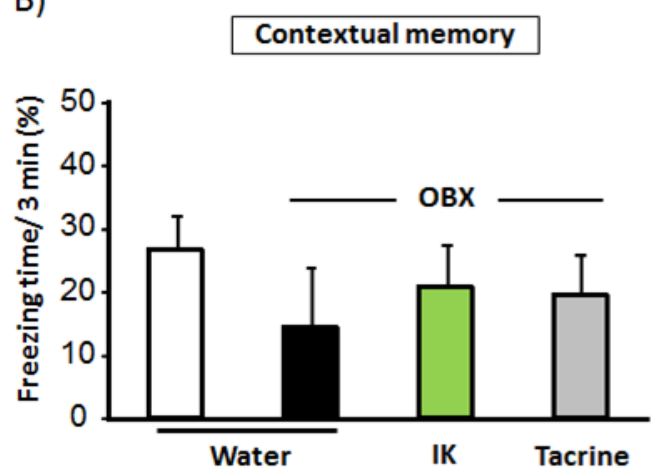

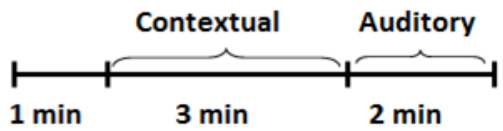

C)

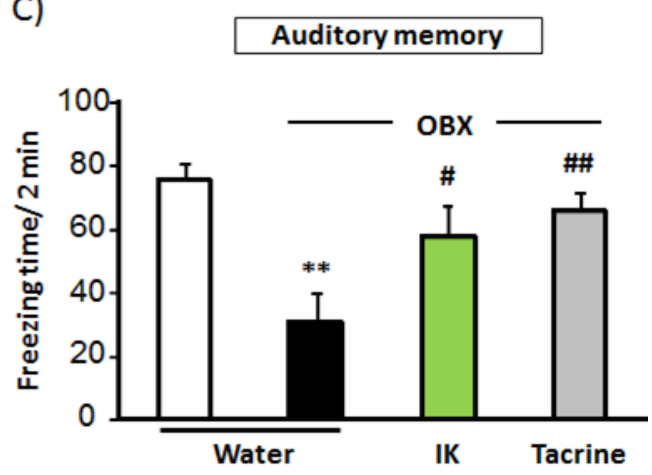

Figure 4. IK saponin ameliorated long term memory deficit in OBX mice. ${ }^{* *} \mathrm{P}<0.01$ vs. sham group; $\# \mathrm{P}<0.05, \# \# \mathrm{P}<0.01$ vs. water-treated OBX group $(\mathrm{n}=9-12)$

treatment with the primary antibodies, membranes were incubated with HRP-conjugated secondary antibodies: anti-rabbit and anti-mouse IgG (1:5000, Thermo Scientific, IL, USA), at $25^{\circ} \mathrm{C}$ for $1 \mathrm{~h}$. Antibody binding was detected using ECL Western blotting detection reagents (Thermo Scientific, IL, USA) and analyzed by AE-9300H EzCapture MG (ATTO).

\section{Data analysis}

The data are expressed as the mean \pm SEM. The data were analyzed by paired and unpaired Student's t-test or one-way analysis of variance (ANOVA) followed by a post hoc multiple comparison tests (Student-Newman-Keul's method) as appropriate. Differences of $\mathrm{P}<0.05$ were considered as significant. The analysis was conducted using SigmaStat ${ }^{\circledR}$ ver. 3.5 (SYSTAT Software Inc., Richmond, CA, USA).

\section{RESULT AND DISCUSSION}

IK extract ameliorates OBX-induced object recognition memory deficit in mice

In this study, we employed OBX mice to analysis the anti-AD effect of IK extract. In fact, OBX in rodent has been widely used as a model of cognitive and emotional dysfunction including Ahzheimer's disease (AD) [6,7,9]. Indeed, the impairment of olfactory perceptual acuity is observed at the early stage of AD [10] and in mild cognitive disorder (MCI) patients [11] as well as in a transgenic $\mathrm{AD}$ model of mice over-expressing a mutant form of the human amyloid- $\beta$ - precursor protein [11].

We employed the ORT to analysis the object recognition memory of mice, a non-spatial shortterm memory. As shown in Figure 2B, in the sample phase trials, none of the animal group showed significant differences in time spent exploring each identical object. In the test phase trial, the sham group spent significantly longer time exploring the novel object than exploring the familiar object $(\mathrm{p}<0.01)$. Vehicle-treated OBX mice showed no preference for the novel object, indicating an object recognition memory deficit. IK extract and tacrinetreated OBX mice significantly spent a longer time for exploring the novel object than that for the familiar one $(\mathrm{p}<0.05)$ (Figure 2C).

\section{IK extract attenuated OBX-induced spatial short-term memory in the manner reversed by scopolamine}

The modified Y maze test was conducted to evaluate the effect of IK extract on spatial shortterm memory in OBX mice. As shown in Figure 3B, sham mice preferred to visit the new arm compared to two familiar arms (higher than the chance level 33.3\%). Vehicle-treated OBX mice significantly spent a shorter time in the new arm compared to sham operation group mice $(\mathrm{p}<0.05)$, indicating the impairment of spatial short-term memory. OBX mice treated with IK extract or 


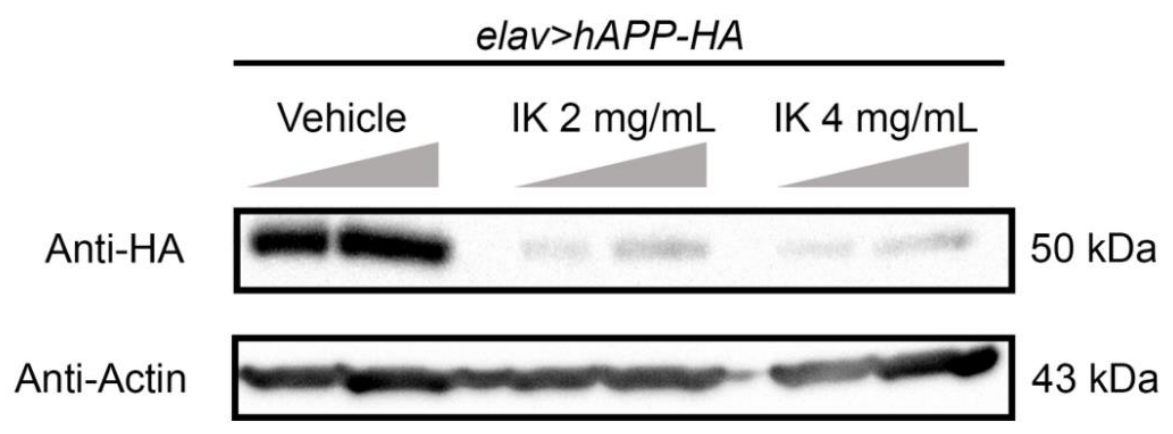

Figure 5. APP expression level at the concentrations of 2 and $4 \mathrm{mg} / \mathrm{mL}$ of IK extract.

acrine significantly spent a longer time exploring the new arm compared to vehicle-treated OBX mice $(\mathrm{p}<0.05)$.

At day 31 after OBX operation, the mice were subjected to modified Y maze to test the involvement of muscarinic receptor in the action of IK extract. Again, the percentage of time in new arm of sham animals was higher than that of vehicle-treated OBX mice. However, the IK and tacrine-treated OBX mice showed a no preference for exploring the new after injecting a single dose of scopolamine $(1.0 \mathrm{mg} / \mathrm{kg}$, i.p), a muscarinic receptor antagonist (Figure 3C).

\section{IK extract ameliorated long-term memory deficit in OBX mice}

In this study, long-term memories of mice were analyzed using fear conditioning test. In this test, we evaluated contextual memory and auditory memory of mice as freezing responses of the mice to the contextual and auditory stimuli, respectively. Contextual memory and auditory memory elucidated in fear conditioning test depend on the hippocampal function and the hippocampus plus amygdala function, respectively [8]. In the conditional fear memory test, no significant difference on the freezing time was observed among animal groups (Figure 4B). Figure $4 \mathrm{C}$ showed that freezing response to the auditory stimuli of vehicle-treated OBX were marked decreased compared with those of sham mice $(\mathrm{p}<0.01)$. However, IK extract and tacrine -treated OBX mice increased the freezing time in this auditory test compared to vehicle-treated -OBX mice $(\mathrm{p}<0.05)$.

Furthermore, the treatment of IK extract was suppressed the $A \beta$ production in adult brain flies by using western blotting with anti APP-HA antibody (Figure 5). IK extract at concentration of 2 and $4 \mathrm{mg} / \mathrm{ml}$ had tendency to reduce the expression level (about 5 and 10 times) with $\mathrm{p}<$ 0.05 and 0.01 correspondingly.
In this study, we demonstrated that OBX mice impaired non-spatial short-term, spatial working memory and long-term memory in ORT test, modified Y-maze test and fear conditioning test, respectively. These findings agree with some previous report $[6,7,14]$. These memory performances of OBX mice were attenuated by IK extract and tacrine, a cholinesterase inhibitor. Interestingly, the beneficial effect of IK and tacrine on OBX-induced spatial short-term memory elucidated in the modified Y-maze test was abolished by scopolamine, a muscarinic receptor antagonist. It has been reported that central cholinergic and glutamatergic systems are implicated in short-term spatial working memory performance elucidated in the modified Y-maze test. Moreover, facilitation of the cholinergic systems by acetylcholinesterase inhibitors, donepezil and THA, is able to improve the memory performance impaired by OBX, the muscarinic receptor antagonist scopolamine and the antiNMDA receptor antagonist MK-801 [6, 7,15]. Taken together, it may infer that IK treatment protects central cholinergic systems from OBXinduced neuronal damage and/or facilitates the systems and thereby improves short-term memory performance in OBX animals.

Kim et al., [3] previously reported that IK ethanol exerts a neuroprotective effect against $A \beta$ (25-35)-induced neurotoxicity in vitro and in vivo. The neuroprotection provided by IK against $A \beta$ induced memory impairment via inhibition of apoptotic neuronal cell death triggered by oxidative stress and p-tau formation. Since OBX is also induces elevation of $A \beta$ level [12], it can be consider that the anti-apoptosis mechanism may contribute to anti-dementia effect of the IK extract in OBX mice.

\section{CONCLUSION}

The present study indicated that IK extract improves short-term and long-term memory 
disturbances in OBX mice and that muscarinic receptor may play a role on these actions. This finding suggested that treatment of IK may be beneficial for prevention/therapy of dementia. In addition, our results also indicated that IK extract reduces the expression of APP protein in Alzheimer's disease flies' brain.

\section{REFERENCES}

Li L, Xu JL, Ma ZL, Dong MJ, Peng Y, et al., 2013. 'The large-leaved Kudingcha (Ilex latifolia Thunb and Ilex kudingcha C.J. Tseng): a traditional Chinese tea with plentiful secondary metabolites and potential biological activities'. J Nat Med 67: 425-437.

Kim JY, Jeong HY, Lee HK, Yoo JK, Bae.K., et al., 2011. 'Protective effect of Ilex latifolia, a major component of "kudingcha", against transient focal ischemia-induced neuronal damage in rats'. J Ethnopharmacol: 558-564.

Kim YJ, Lee KL, Jang YJ, 2015. 'Ilex latifolia prevents amyloid $\beta$ protein (25-35)-induced memory impairment by inhibiting apoptosis and tau phosphorylation in mice'. J Med Food 18: 1317-1326.

Kim JY, Lee HK, Hwang BY, Kim SH, Yoo JK, et al., 2012. 'Neuroprotection of Ilex latifolia and caffeoylquinic acid derivatives against excitotoxic and hypoxic damage of cultured rat cortical neurons'. Arch Pharmacal Res 35: 1115-1122.

Le TX, Nguyen HA, Pham TNH, Nguyen VT, Nguyen $\mathrm{TP}$, et al., 2015. 'Antidepressant-like effect of Ocimum sanctum in Olfactory Bulbectomized mice'. Tạp chí dược liệu 20 311-316.

Le XT, Pham HT, Do PT, Fujiwara H, Tanaka K, et al., 2013. 'Bacopa monnieri ameliorates memory deficits in olfactory bulbectomized mice: possible involvement of glutamatergic and cholinergic systems'. Neurochem Res 38: 2201-2215.

Yamada M, Hayashida M, Zhao Q, Shibahara N, Tanaka K, et al., 2011. 'Ameliorative effects of yokukansan on learning and memory deficits in olfactory bulbectomized mice'. Journal of Ethnopharmacology 135: 737746.

Phillips RG, LeDoux JE, 1992. 'Differential contribution of amygdala and hippocampus to cued and contextual fear conditioning'. Behav Neurosci 106: 274-285.

Oba A, Nakagawasai O, Onogi H, Nemoto W, Yaoita $\mathrm{F}$, et al., 2013. 'Chronic fluvoxamine treatment changes 5 -HT(2A/2C) receptormediated behavior in olfactory bulbectomized mice'. Life Sci 92: 119-124.

Bahar-Fuchs A, Chetelat G, Villemagne VL, Moss S, Pike K, et al., 2010. 'Olfactory deficits and amyloid-? burden in Alzheimer's disease, mild cognitive impairment, and healthy aging: a PiB PET study'. J Alzheimers Dis 22: 1081-1087.

Bahar-Fuchs A, Moss S, Rowe C, Savage G, 2010. 'Olfactory performance in $\mathrm{AD}, \mathrm{aMCI}$, and healthy ageing: a unirhinal approach'. Chemical Senses 35: 855-862.

Aleksandrova IY, Kuvichkin VV, Kashparov IA, Medvinskaya NI, Nesterova IV, et al., 2004. 'Increased level of beta-amyloid in the brain of bulbectomized mice'. Biochemistry (Mosc) 69: 176-180.

Hozumi S, Nakagawasai O, Tan-No K, Niijima F, Yamadera F, et al., 2003. Characteristics of changes in cholinergic function and impairment of learning and memory-related behavior induced by olfactory bulbectomy. Behavioural Brain Res 138: 9-15.

Sithisarn P, Rojsanga P, Jarikasem S, Tanaka K, Matsumoto K, 2013. 'Ameliorative effects of Acanthopanax trifoliatus on cognitive and emotional deficits in olfactory bulbectomized mice: An animal model of depression and cognitive deficits'. Evid Based Complement Alternat Med 2013: 701956.

Csernansky JG, Martin M, Shah R, Bertchume A, Colvin J, et al., 2005. 'Cholinesterase inhibitors ameliorate behavioral deficits induced by MK-801 in mice'. Neuropsychopharmacol 30: 2135-2143. 